\title{
Traditional Enterprise Business Intelligence Software Compared to Soft- ware as a Service Business Intelligence
}

\author{
Marian Pompiliu CRISTESCU \\ "Lucian Blaga" University of Sibiu, Romania \\ marian.cristescu@ulbsibiu.ro
}

The focus of this paper is on the one hand on the importance of business intelligence in large and medium-sized businesses and on the other side on the evaluation and implementation of Business Intelligence. It shows how companies make better and faster decisions regarding their customers, partners and operations by converting data into valuable business information. The paper describes how to bring business intelligence information, people and technology together to help to create a successful business strategy. The conclusion of the paper is the assessment of the possibility of business intelligence to develop projects in large and medium-sized companies and the discussion of the possible alternatives with respect to the different functions.

Keywords: Business Intelligence Solutions, Business Processes, Data Warehouse, Cloud Data, Process

\section{Introduction}

Business Intelligence is a broad field of study. The major thrust of business intelligence theory analyzes certain factors to make highquality decisions. These factors include customers, competitors, business partners, economic environment and internal operations. Business Intelligence has the power to change the way people work, so that enterprises can compete more effectively and efficiently.

A successful business intelligence solutions and successful implementation holds the key to the technological innovations together with the people, processes and culture of an organization to achieve a competitive and profitable BI strategy.

Business Intelligence (BI) is the process of acquiring operational data and the use of this data in order to make the best business decisions. BI software takes the information bulk of the operational data sources of an enterprise, integrates and analyzes them and presents timely verdicts in the form of reports, alerts and dashboards.

These reports, alerts and dashboards provide a more accurate and actionable view of the situation of a company.

The success of a BI solution is ultimately in how well it helps both business and technical users in an organization to meet their missioncritical objectives: meeting or exceeding the revenue figures, looking for ways to reduce costs across the organization and maximize profitability by identifying the most profitable customers, products, services or programs. In fact, a BI solution should be able to identify otherwise unknown situations.

Within an organization a BI solution helps users meet the following critical business objectives: meeting or exceeding the revenue figures, maximizing profitability by identifying the most profitable programs, looking for ways to reduce costs across the organization, creating a 360-degree view directed to customers.

A business intelligence solution allows business teams the correlation of data across the enterprise to understand and to synthesize comprehensive analysis of the results, trends and expected results in order to understand the implications of this insight on corporate goals. BI users have an understanding of what is happening and what future actions need to be taken.

\section{Business Intelligence Solutions}

Business intelligence (BI) solutions have already proven their worth a lot of times in the past. The reason for their popularity can be explained by various new developments.

Especially the following trends play a major role: on the one hand, the emergence of new 
valuable and relevant data sources, on the other hand, a new generation of BI visualization tools based on HTML5 and JavaScript, and finally the removal of many traditional ERP functionalities and the growth of new enterprise capabilities in the cloud.

Here are a few questions that can be easily answered by BI:

\section{Sales and marketing issues:}

$>$ Which customers does a company want to target group?

$>$ What caused the change in the pipeline?

$>$ Which are a company's most profitable campaigns per region?

$>$ What's the turnover after an announcement of a new product release?

What is the most profitable source of revenue and how has it changed over time?

\section{Operational Questions}

$>$ Which vendors have the best provision of time and budget?

$>$ How much additional staff is needed per store during the holidays?

$>$ What order fulfillment process is highly inefficient?

\section{Financial Questions}

$>$ What are the costs for new products?

$>$ What is the expected annual profit / loss on the basis of current marketing and sales activities predictions?

$>$ What are the forecasts compared to the annual plan?

$>$ What are the current trends in cash flow, accounts payable and accounts receivable, and how are they comparing to the plan?

\section{Business Performance Questions}

What are the main risk factors affecting the company's ability to meet the annual profit goals?

$>$ Shall we expand the company internationally and, if so, what geographical areas we should first target?

\section{Traditional Business Intelligence Enter- prise Software}

Traditional business intelligence solutions are delivered as enterprise software, meaning that the software solutions are installed and supported on the premises of its own IT team. The software is expensive and often requires hundreds of thousands or millions of dollars in upfront costs.

The implementation of the software is also expensive, in addition to the costs with the employees, it often requires to purchase additional hardware for the software, such as hardware servers. Maintenance and support costs, however, are lower than investment costs.

Traditional BI solutions are delivered expensive. SaaS BI solutions resolve from the cost burden, time requirements and but have limitations of traditional solutions.

Moreover, a single BI solution could actually require the implementation and purchase together with the integration of multiple software components, such as software for extracting data, data integration, storage, analysis, and reporting. Here is a diagram of a typical traditional business intelligence solution implementation (Figure 1). 


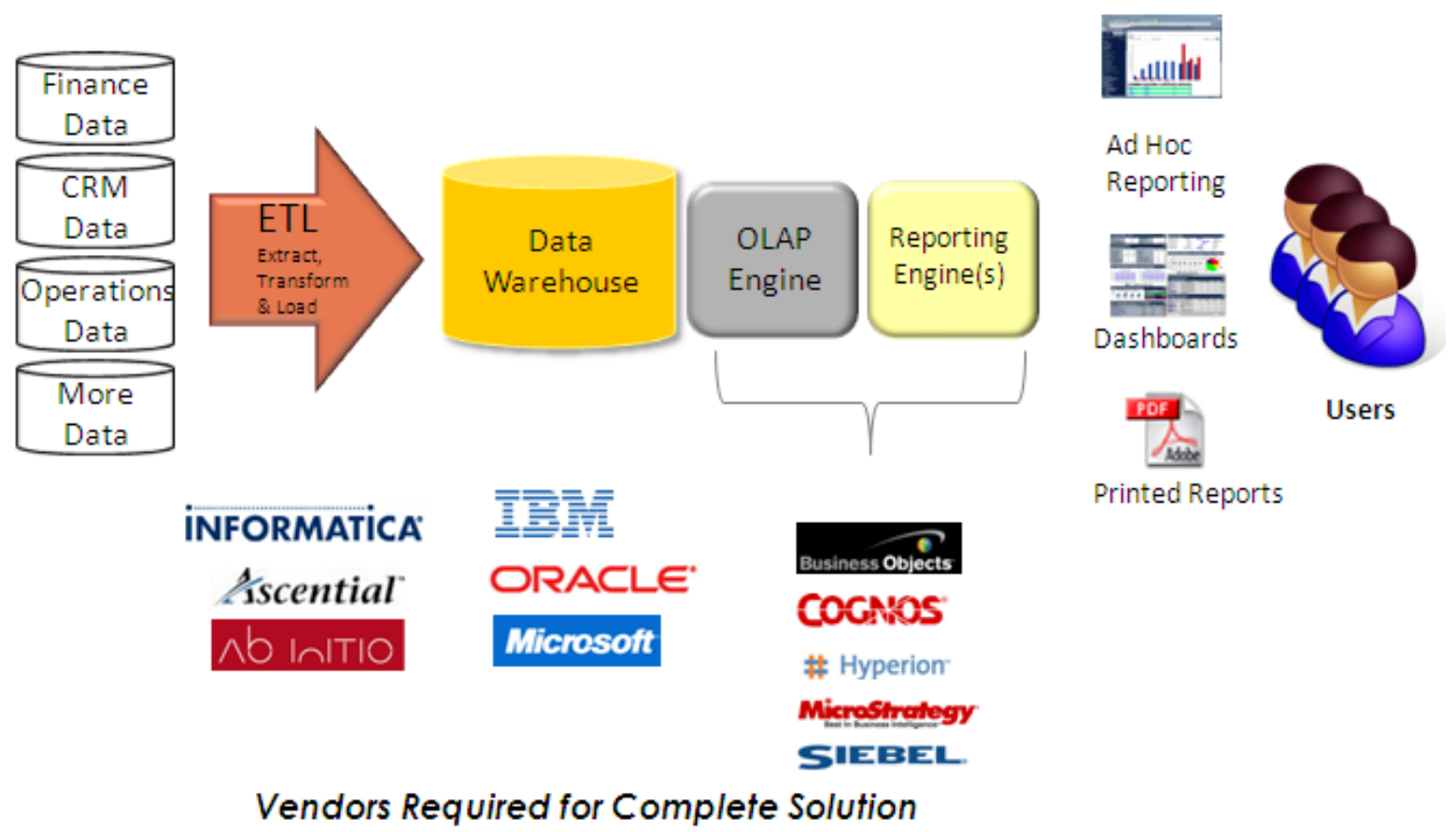

Fig. 1. Traditional BI solution implementation

Companies appreciate the value of the analysis they can get from traditional BI solutions, so that they are willing to invest in time, software, hardware and human resources. But this high cost discourages the small and medium enterprises, as they can find it difficult to go ahead with such a solution. Some recent analyzes conducted by independent analysts' study shows the challenges of traditional BI deployment software companies confront.

\section{Software as a Service (SaaS) or On-De- mand Business Intelligence}

Software as a Service Business Intelligence (BI known as SaaS or On-Demand BI) was created in response to the complexity, resource drain, long delays and prohibitive cost of traditional enterprise software. Instead the purchase, integration and implementation of software itself, SaaS BI customers buy BI capabilities as a service from a dealer.

The software does not need to be implemented and maintained on the premises of the company, but can run on servers in a remote location supported by the sellers. The company accesses the services via a secure Internet connection. Salesforce.com, NetSuite and RightNow are examples of successful SaaS solutions for Customer Relationship Management.
SaaS solutions offer significant advantages over traditional software for small and medium enterprises. SaaS requires to implement weeks or months, rather than years, as in the traditional solutions BI. The upfront investment costs are drastically reduced, the majority of the risk is removed - there is no software to buy, without having to buy additional hardware, and less implementation and ongoing costs. SaaS is usually on a subscription basis (usually for a monthly fee) or cheap pay-foruse basis.

For this reason, SaaS solutions are often paid as a business expense instead of issuing costs. One of the keys to the lower cost of SaaS BI is the degree of integration and automation that a SaaS BI vendor uses.

A fully integrated and automated retailer drives the BI Integration for businesses and automates some of the most difficult and timeconsuming components. SaaS also offers ongoing cost and value benefits. SaaS can initially deliver a limited trial project in order to quickly demonstrate the value and effectiveness. Then it can deliver on an iterative basis, so that other data sources and users can be easily added later. This allows companies to first identify the value before making far-reaching commitments and projects with high priority. 
The implementation of other projects is easily performed with less additional effort. SaaS customers are often automatically updated by the provider, which means that individual companies have nothing to do with the time and cost of rollout of software upgrades.

SaaS solutions are more user-friendly than traditional BI solutions that have been built specifically for the use of analytical experts within an IT organization. While the traditional approach allows highly complex analysis, SaaS solutions are often created to serve both business users and expert analysis, so that business users can use the robust reporting and data exploration they need, while experts can handle more complex inquiries.

\section{Traditional Enterprise BI software ver- sus software as a service BI}

The traditional business intelligence solutions are delivered as enterprise software. While these solutions can be effective, they require high investment costs, intensive use of IT resources or 3rd party consulting and hardware investments to get started. Maintenance of the system is also costly and resource-intensive, therefore, these solutions are usually purchased by large companies.

Expansion or enlargement of the solutions is often prohibitively expensive. In recent years, SaaS or on-demand business intelligence solutions have evolved to meet the cost and time for the smaller and medium-sized enterprises. For these reasons, SaaS solutions are gaining new customers in small and medium enterprises.

As one of the latest innovations that is driving these trends, the cloud BI has proven for many companies to be extremely useful. Cloud BI software is suitable for all kinds of businesses because it directly accesses the data exactly where it's mainly generated and stored, namely in the cloud. Thanks to HTML5 and JavaScript support, it can also be used via a browser.

In addition, they can be embedded in a cloud application, so as to enable the most effective way of providing BI within an application Embedded BI. Not least, it provides access to extensive analysis and reporting tools that allow companies to manage their business processes.

Cloud BI software is user-friendly, since they can be embedded into various applications, where they can then be used by users who are not necessarily familiar with BI. The cloud BI thus appeals to a much broader audience than traditional Business Intelligence.

In terms of scaling, the cloud BI is a class of its own. The ability to increase rapidly and keep up with the cloud-development is more important than the ability to run massive BI Server instances.

This new level of usability and scalability makes the Cloud BI extremely interesting for companies, since almost every employee can have access to valuable intelligence - the main advantage of any BI solution.

Business administrators can analyze everyday processes and help their colleagues in other departments in increasing efficiency. But even ordinary people can use cloud BI platforms to streamline standard processes and to make a greater contribution to corporate success.

It is almost needless to say that a cloud-based BI platform can also simplify implementation processes significantly, because the installation and configuration of a BI server is eliminated. A cost-effective cloud BI solution can take a few weeks or even days to implement, comparing to the traditional $\mathrm{BI}$ products that can take several months to implement.

The future will certainly bring us an even better understanding of data-driven applications and Embeddable BI solutions will continue their ascending journey.

\section{The 5 Stages of BI}

Both traditional BI software and SaaS BI solutions include the same five levels below raw data and present it as relevant, actionable data for users. However, when speaking of traditional BI software vs. SaaS BI solutions, each of the five phases may differ in terms of time, cost, complexity and resources.

The five stages are:

1. Data

2. The ETL (Extract, Transform, and Load) engine 
3. Data Warehousing

4. Analytic Engine

5. Presentation Layer

The first four stages of the BI process include the "background" processes and are traditionally driven by the technical team. Most business users interact with the presentation layer. The background of BI consists of entering, integration and analysis of data. Traditional enterprise software and SaaS solutions take different approaches to the background processes and require different means and timeframe.

\section{- Level 1: Data}

The first step in the BI process is to determine and define which data will be loaded and analyzed in the system. A key question at this stage is whether all data is defined in advance or whether to identify an iterative basis.

Traditional method: The entire data structure must be set; the companies must decide which data they want to use before starting the rest of the background processes in advance.

Integrated SaaS method: Companies can start with the available data without determining the data sources in advance. Then additional data sources can be added on a continuous basis.

\section{- Level 2: The ETL Engine}

The ETL (Extract, Transform, and Load) engine is the movement of the source data to the data warehouse. If this does not work properly, the BI solution cannot be effective.

Traditional method: Technical teams take manual data sources to define tables and assign them subsequently in a data warehouse production environment. This process is repeated for each BI initiative within the organization.

Integrated SaaS Method: the entire process can be automated to create production and warehouse tables. There are two other important issues at this stage:

What kind of data is entered into the system? The data types that can be used by BI solutions should be all the information that could be useful for a business user. A BI solution should allow companies or groups to integrate files of large size with each application that can export data:

- Data extracts from the operational applications, such as CRM or ERP applications;

- SQL, Oracle or other relational databases;

- Flat File databases;

- Comma-separated files (default "generic" file format);

- Excel or other spreadsheet software;

Optimally, the bi solution should have an automated data updating system. Otherwise, the data must be uploaded and updated manually. Business intelligence vendors should be able to offer the development of an integration plan directly or through a partner.

\section{- Level 3: Data Warehousing}

Data Warehouse combine and retain electronic data - either in a large system or multiple connected systems, so that the data can be retrieved and analyzed.

Traditional method: Traditionally, this is a very frustrating stage for companies because they are forced to select between two suboptimal approaches: Multiple Data Marts and storage charged by several ETL processes, which reduces the scope of an application, or an Enterprise Data Warehouse (EDW), which provides scale but limits the flexibility of providing new analytical applications.

Integrated SaaS Method: Data warehouses are created for each business intelligence project or application. There is no dependence on a single EDW approach.

\section{- Level 4: Analytic Engine}

After all the data is in the system, the next step is to perform the analysis. Normally, this phase requires considerable technical resources to analyze the data warehouse of the company. In this phase, SaaS BI solutions can offer an automated approach to the analysis and move them online with an integrated OLAP (On-Line Analytical Processing) engine.

Traditional method: the IT team developed another set of metadata that move between universes, Subjects and cubes or create custom cubes for analysis. 
Integrated SaaS method: metric and dimensional stability of the metadata can be automatically generated and maintained.

\section{- Stage 5: Presentation Layer}

BI solutions include tools for the presentation of results from the analyzed data:

\section{Interactive Dashboards}

A dashboard is a series of high-level reports on key figures typically for executives. There may be multiple reports on a single dashboard. With a dashboard, users can get at a glance understanding of key trends and Optometry. Dashboards can be personalized for each individual in an organization, a salesperson or Frontline manager to a manager on middle management or leadership.

An interactive dashboard enables users to filter information, to analyze trends and results, to lower or tear deeper into a more detailed analysis of the data. This means that by clicking on the reports or results, they can find detailed information on what lead to those results.

Customizable reports that present high-level results or allow access to specific details. Most BI systems come with either report templates and / or offer the ability to create and customize reports.

Warning alerts that notify the users about an upcoming event, changes to data, or that new data must be entered into the system.

Traditional method: reporting packages must be implemented by multiple solutions, since only some of them offer perfect coverage. The IT team must create and publish in the organization unique dashboards and reports for any other type of user.

Integrated SaaS Method: a fully integrated reporting package is ideal, so it does not need to be integrated across multiple systems. Advanced reporting techniques, such as banded reports, drag and drop formatting and pixelperfect reports, all contained in a solution.

As in all markets, there are a host of different companies that are active in this area that offer BI services either in the traditional way of an on premise BI system or via a cloud base BI software but also hybrid solutions managed by both on premise and cloud servers and data.
The market offers enough space of development for both big and small companies and rewards a lot of the new comers in the market, due to the need of constructive and effective ideas to perform the needed tasks.

One of the markets pictures of the available software is offered by Gartner in the Reviews for BI and Analytics Platforms or in the yearly articles of the Magic Quadrant for Business Intelligence and Analytics Platforms. If we look at the different stages of BI and try to identify them in a few of the companies listed in the articles above, we can see some good examples of vendors offering innovative new approaches as well as the "veterans" in this field.

Looking at the first stage of BI, the data sourcing plays an important role in the early steps of a successful BI solution. Microsoft is positioned in the Leaders quadrant, with strong uptake of the latest release, major product improvements, an increase in sales and marketing awareness efforts, new leadership and a clearer, more visionary product roadmap. Microsoft is one of the important players in the BI field and its continuous focus on expanding the variety of the data sources it supports, has paid out.

For example, Microsoft now has prebuilt connectors (and content) to Facebook, Salesforce, Dynamics CRM, Google Analytics, Zendesk, Marketo and more. A data source is the true origin of the data that users are investigating in their reports and dashboards. This may, for example, be an online service such as Google Analytics or QuickBooks, a database in the cloud such as Azure SQL Database or a database or file on a local computer or server in their own organization.

A dataset is automatically created in Power BI, when you "retrieve data" to initiate a connection and upload data from a content pack or connect to a live data source. Microsoft continues to support the original Excel-based add-ins that made up the first Power BI release: Power Query, Power Pivot, Power View and Power Map.

The Excel-based add-ins are positioned primarily for customers who need an on-premises deployment (and become native in Office 
2016). Power BI 2.x offers both desktopbased authoring and browser-based authoring, with applications shared in the cloud. New in this release is hybrid connectivity to on-premises data sources; meaning that not all data must first be pushed and loaded into the Microsoft Azure cloud.

An interesting new player, that has brought the attention upon himself with its implementations, is ClearStory Data (http://www.clearstorydata.com/). In the area of The ETL Engine and Data Warehousing they have brought a fast way of integrating data from mixed sources, local as well as cloud data in addition to the ability to even use enterprise as well as complex industry syndication data (such as IMS, Nielson, IRI).

Citing the above sources ClearStory Data has excelled in its possibility to quickly gather the data and make it available to its users. This is not the only company allowing for this kind of integration but it is one of the companies that brought an interesting new way of perfuming it faster.

In terms of an innovative combination of automated smart pattern discovery and identification of insights, BeyondCore was ranked in the top quartile of all Magic Quadrant vendors for market understanding and also for both underlying components - complexity of analysis and ease of use. Being still a small vendor, BeyondCore is preferred by users for its ease of use for end users, functionality and implementation cost and effort. Both sales experience and support has made this small company a preferred solution for many other companies.

The algorithms and code that BeyondCore uses to render results is completely transparent to data scientists to validate findings and export and extend the model; this is critical to BeyondCore avoiding being labeled as a "black box" solution, a label that has negative connotations in the market. BeyondCore has partnered with Microsoft Analyst for Office, which integrates into Word, Excel, PowerPoint or Outlook and allows ten analyses per month for free on datasets in Excel or Microsoft SQL. A premium service allows unlimited analyses as well as the ability to query larger datasets, including big data ones in Hadoop, SAP HANA or Oracle.

A good example of a growing player in the BI market is Salesforce through its interesting approach to the Presentation Layer. They offer a natively mobile platform, which comes with a built in collaboration component through Salesforce Chatter, that allows virtually anyone to get started with the reports and also creates a simple way of communication among the different levels of the software within the business.

Their new vision in this area allows users to use both cloud and on premise data when doing the analysis and presentation, although the non-cloud data must be entered through partners and cannot be used directly. All in all Salesforce appears to be one of the companies targeting an easy to use way of getting access to the relevant data to both power users as well as the users just starting down this road.

The above does not mean that these examples are the best in their areas or that they are the only ones bringing inventions to these parts of the BI system, the "veteran" players at this table like Microsoft, SAP or SaaS are all bringing new an interesting ideas to this evolving service as a whole.

We can easily mention Microsoft's new approach to pricing, being priced almost with the lowest cost per user or SAP's excellent surveys on business application integration, but this was not the idea of this article and the examples above provide a small insight on the different approaches and options available within this market.

\section{Conclusion}

Business Intelligence solutions help organizations gain actionable insights from their business data in order to achieve business goals. Most operational groups already have some ways to use some of the information within their areas, which are typically represented by tables, databases or limited coverage in an operational solution, like a customer loyalty management solution. However, business intelligence solutions help large companies to easily obtain insight on the specific points of 
interest, by making the analysis and integration of larger amounts of data from multiple areas of the company possible, and allows them to perform complex calculations and have an easier way distribute compelling reports and dashboards.

Business intelligence solutions can be a good option for companies either in the traditional way or as integrated SaaS. The powerful, traditional business intelligence software can require significant upfront costs, lengthy implementations and high resource requirements for hardware and IT services. SaaS business intelligence solutions are a modern and flexible approach, with considerable value advantage, but they are less flexible compared to traditional software. SaaS BI can be implemented quickly, in weeks rather than months, have lower investment costs and significantly lower need of resources, which makes it less complex than traditional BI solutions.

Business intelligence solutions have a growing demand, because the available data is rapidly expanding and companies are looking for analysis and insight that can bring them a competitive advantage in a challenging economic environment. Business intelligence aims to support better business decisions or a better decision making process.

Through the current growing demand, we can clearly see the evolution of the SaaS solution that allows a broader selection of companies to access the benefits of a BI solution. Moving from a traditional market for the BI solutions one can observe that even the smaller companies can now benefit from the power of a BI solution and by using this, they gain a competitive advantage over other companies in the same field of operations.

In a market rapidly driven by fast and accurate results, the SaaS advantage allows a usage of $\mathrm{BI}$ in virtually any area that can deliver a workable set of data and can help companies in all fields get an overview on the current status of the business and offer a valuable set of information on how to either expand or grow the current business within the targeted market.
Looking at the current market, we can see the parallel evolution of both available solutions for BI, an evolution that is benefiting both small and big companies alike. From here on an even bigger evolution is foreseeable as more and more companies will understand the advantage a BI solution can offer for the development and management of the business and through this usage also drive the evolution of both solutions in a time where accurate data analysis and explanations provides an edge in any area or business.

\section{References}

[1] Business Intelligence - ein Überblick, Arbeitspapier, an der Universität ErlangenNürnberg 2/2002.

[2] P. Azevedo, G. Brosius, S. Dehnert, B. Neumann, B. Scheerer, "Business Intelligence und Reporting mit Microsoft SQL Server 2005", Microsoft Press, 2006.

[3] P. Keller, „Was macht BusinessIntelligence-Projekte erfolgreich?" $P D F$ Dokument zur CeBIT 2005, von BARC, 2005.

[4] H.G. Kemper, W. Mehanna, C. Unger, „Business Intelligence Grundlagen und praktische

Anwendungen“,Vieweg, 2004.

[4] Microsoft Corporation: "Online Documentation SQL Server 2008", Microsoft Corpora

tion, 2010.

[5] P. Rita, L. Sallam, J. Richardson, J.

Hagerty, B. Hostmann. "Magic Quadrant for Busi -

ness Intelligence Platforms", 2011.

[6] H. Schrödl, ,,Business Intelligence mit

Microsoft SQL Server 2005“, Hanser, 2006.

[7] M. Schultz, B. Martin, J. Knuth, V. Pruß, „Microsoft SQL Server 2008 Reporting Services - Das Praxisbuch“, Microsoft Press, 2008

[8] Azevedo, G. Brosius, S. Dehnert, B. Neumann, B. Scheerer, "Business Intelligence und Reporting mit Microsoft SQL Server 2005", Microsoft Press, 2006. 


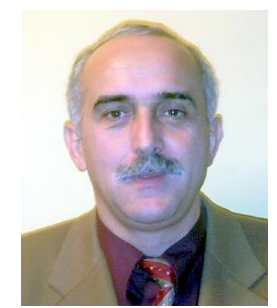

Marian-Pompiliu CRISTESCU has graduated the Faculty of Planning and Economic Cybernetics in 1985, he holds a PhD diploma in Economics from 2003, obtained at the Faculty of Cybernetics, Statistic and Economic Informatics from the Academy of Economic Studies in Bucharest. In 1991 he joined the university teaching system, going through all didactic positions starting with teaching assistant, lecturer in 2001 and assistant professor since 2008. Presently, he is an associate professor in Economic Informatics at the Faculty of Economic Sciences - "Lucian Blaga" University of Sibiu. He is the author of 14 books and over 100 scientific articles in the field of software quality, programming environments, data bases and economic informatics systems. He is equally focused on software development, being the author or co-author of over 45 programming systems for economic management. He has received many diplomas to certify his achievements in the scientific research domain. He has participated as a project director or as a leading team member at 12 research contracts. 